\title{
Gauge Invariant Formulations of Dicke-Preparata Super-Radiant Models
}

\author{
S. Somu and A. Widom \\ Physics Department, Northeastern University, Boston MA 02115 \\ Y.N. Srivastava \\ Physics Department 8 INFN, University of Perugia, Perugia Italy

$$
\mu=\sum_{a=1}^{N} q_{a} \mathbf{r}_{a}
$$

In a gauge invariant formulation of the molecular electric dipole-photon interaction, the rigorous coupling is strictly linear in the photon creation and photon annihilation operators. The linear coupling allows for a super-radiant phase transition as in the Hepp-Lieb formulation. A previous notion of a quadratic-coupling "no-go theorem" for super-radiance is incorrect. Also incorrect is a previous assertion that the dipole-photon coupling has absolutely no effect on the thermal equations of state. These dubious assertions were based on incorrect canonical transformations which eliminated the electric field (and thereby eliminated the dipole-photon interaction) which is neither mathematically nor physically consistent. The correct form of the canonical transformations are given in this work which allows for the physical reality of super-radiant condensed matter phases.

PACS: 78.60.Kn, 78.60.Fi, 78.70.-g

\section{INTRODUCTION}

In an early paper on the interaction between radiation and molecular dipole moments [1], Dicke formulated a model which was later shown to exhibit a super-radiant phase transition [2, 3]. The notion that such phase transitions should exist in condensed matter systems has been investigated in a series of papers by Preparata

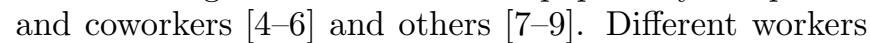
have come to somewhat different conclusions concerning super-radiant phase transitions 10 19. Some doubt has been expressed 20 24] concerning the physical laboratory reality of super-radiant phase transition. The mathematical issues are as follows: (i) It appears, at first glance, that quadratic terms (in photon creation and annihilation operators) enter into the model via quadratic terms in the vector potential A. (ii) The quadratic terms in the "corrected Dicke model" appear to destroy the super-radiant phase transition.

Our purpose is to show that if the dipole-field interaction is treated in a gauge invariant manner [25 27] then the interaction is strictly linear in the electric field E. Thus, quadratic terms are absent for purely electric dipole-photon interactions [28]. These considerations render likely the physical reality of condensed matter super-radiant phase transitions.

To see what is involved, let us first consider a single molecule with an electric dipole moment $\mu$ given by where $q_{a}$ and $\mathbf{r}_{a}$ denote respectively, the charge and position of the $a^{t h}$ particle. Suppose that the dipole moment interacts with a classical electric field which is uniform in space, but not in time

$$
\mathbf{E}(t)=-\frac{1}{c}\left(\frac{d \mathbf{A}(t)}{d t}\right) .
$$

The molecule will have a time dependent Hamiltonian of the form

$$
H_{m o l}(t)=\sum_{a=1}^{N} K_{a}\left(\mathbf{p}_{a}-\frac{q_{a}}{c} \mathbf{A}(t)\right)+V\left(\mathbf{r}_{1}, \ldots, \mathbf{r}_{N}\right),
$$

where $K_{a}\left(\mathbf{p}_{a}\right)=\left(\mathbf{p}_{a}^{2} / 2 m_{a}\right)$ is the kinetic energy of $a^{t h}$ particle in the molecule and $V\left(\mathbf{r}_{1}, \ldots, \mathbf{r}_{N}\right)$ is the internal Coulomb energy of the molecule. Employing the unitary operator

$$
U(t)=e^{i(\mu \cdot \mathbf{A}(t) / \hbar c)}
$$

in the time dependent canonical transformation

$$
\mathcal{H}_{\text {mol }}(t)=U^{\dagger}(t) H_{\text {mol }}(t) U(t)-i \hbar U^{\dagger}(t) \frac{d U(t)}{d t}
$$

yields

$$
\mathcal{H}_{\text {mol }}(t)=\sum_{a=1}^{N} K_{a}\left(\mathbf{p}_{a}\right)+V\left(\mathbf{r}_{1}, \ldots, \mathbf{r}_{N}\right)-\mu \cdot \mathbf{E}(t) .
$$

For completeness of presentation, a derivation of Eq.(5) is given in Appendix A.

Note: For classical electric fields of the form in Eq.(2), the Hamiltonians in Eqs.(3) and (6) are rigorously equivalent. Furthermore, the Hamiltonian in Eq.(3) depends on linear and quadratic terms in the vector potential $\mathbf{A}(t)$. On the other hand, the Hamiltonian in Eq.(6) depends only on the electric field $\mathbf{E}(t)$ and in a strictly gauge invariant and linear fashion.

In the work which follows, both the charged particles and the electromagnetic field will be treated using quantum mechanics. In Sec.II we discuss a single molecule in the presence of the quantized electromagnetic field. It is proved for the gauge invariant description of the dipole interactions that the coupling between the electromagnetic field and the molecule is linear in photon creation 
and annihilation operators The results for $\mathcal{N}$ molecules are discussed in Sec.III. The resulting Hamiltonian has four terms: (i) a sum of single molecule Hamiltonians, (ii) a sum of screened Coulomb interaction potentials between neighboring molecules, (iii) the radiation field energy and (most importantly) (iv) a strictly linear coupling between the dipole moments and the electric field. DickePreparata models are defined and explored in Sec.IV. In the concluding Sec.V, the importance of the superradiant phase transition associated with linear couplings will be discussed. The errors by some previous workers who falsely "voided" the super-radiant phase transition will be discussed in detail.

\section{PHOTON-DIPOLE INTERACTIONS}

Consider, as in Sec.I, a single molecule having a total number of $N$ charged particles with an electric dipole moment $\mu$ as in Eq.(1). The dipole is considered to interact with a quantized electromagnetic field (photons) A and/or $\mathbf{E}$ which is again uniform in space. The Hamiltonian for the photons is given by

$$
H_{\text {rad }}=\sum_{k} \hbar \omega_{k} a_{k}^{\dagger} a_{k}
$$

where

$$
\left[a_{k^{\prime}}, a_{k}^{\dagger}\right]=\delta_{k k^{\prime}}
$$

Furthermore, for a "quantization box" of volume $\Omega$,

$$
\mathbf{A}=c \sqrt{\frac{2 \pi \hbar}{\Omega}} \sum_{k} \frac{1}{\sqrt{\omega_{k}}}\left(\mathbf{e}_{k} a_{k}+\mathbf{e}_{k}^{*} a_{k}^{\dagger}\right)
$$

and

$$
\mathbf{E}=i \sqrt{\frac{2 \pi \hbar}{\Omega}} \sum_{k} \sqrt{\omega_{k}}\left(\mathbf{e}_{k} a_{k}-\mathbf{e}_{k}^{*} a_{k}^{\dagger}\right)
$$

The matter Hamiltonian for a single molecule in the electric dipole limit is then

$$
H_{m o l}(\mathbf{A})=\sum_{a=1}^{N} K_{a}\left(\mathbf{p}_{a}-\frac{q_{a}}{c} \mathbf{A}\right)+V\left(\mathbf{r}_{1}, \ldots \mathbf{r}_{N}\right)
$$

In total, the time independent Hamiltonian for the molecule with dipole-quantized electromagnetic field interactions reads

$$
H=H_{\text {rad }}+H_{m o l}(\mathbf{A}),
$$

where $H_{\text {rad }}$ is defined in Eq. (7), and $H_{m o l}(\mathbf{A})$ is defined in Eqs.(9) and (11). In the quantum electrodynamic model, Eq.(2) is replaced by the operator equation

$$
\mathbf{E}=-\left(\frac{i}{\hbar c}\right)[H, \mathbf{A}]
$$

leading to Eq.(10).

Employing the unitary operator

$$
S=e^{i(\mu \cdot \mathbf{A} / \hbar c)}
$$

in the time-independent canonical transformation

$$
\mathcal{H}=S^{\dagger} H S,
$$

yields

$$
\mathcal{H}=H_{\text {rad }}+H_{\text {mol }}(\mathbf{A}=0)-\mu \cdot \mathbf{E}+W_{\text {mol }}
$$

where the "self energy" contribution to the molecule is given by

$$
W_{m o l}=\frac{2 \pi}{\Omega} \sum_{k}\left(\mathbf{e}_{k} \cdot \mu\right)\left(\mathbf{e}_{k}^{*} \cdot \mu\right) .
$$

The derivation of Eq.(16) is given in Appendix B.

Introducing the boson operators $b_{k}=i a_{k}$ and the Hermitian conjugate $b_{k}^{\dagger}=-i a_{k}^{\dagger}$ yields our final Hamiltonian

$$
\begin{aligned}
\mathcal{H}= & \sum_{k} \hbar \omega_{k} b_{k}^{\dagger} b_{k}+\sum_{a=1}^{N} K_{a}\left(\mathbf{p}_{a}\right)+V\left(\mathbf{r}_{1}, \ldots \mathbf{r}_{N}\right) \\
& +\left(\frac{2 \pi}{\Omega}\right) \sum_{k}\left(\mathbf{e}_{k} \cdot \mu\right)\left(\mathbf{e}_{k}^{*} \cdot \mu\right)-\mu \cdot \mathbf{E} .
\end{aligned}
$$

where

$$
\mathbf{E}=\sqrt{\frac{2 \pi \hbar}{\Omega}} \sum_{k} \sqrt{\omega_{k}}\left(\mathbf{e}_{k} b_{k}+\mathbf{e}_{k}^{*} b_{k}^{\dagger}\right)
$$

and

$$
\left[b_{k^{\prime}}, b_{k}^{\dagger}\right]=\delta_{k k^{\prime}}
$$

The central result of this section is the following:

Theorem: For the electric dipole-electromagnetic field interaction, the resulting Hamiltonian is that of free photons plus interaction terms linear in the photon creation and annihilation operators. Quadratic interaction terms rigorously vanish.

Proof: See Eqs.(18) and (19), which were derived solely on the basis of the dipole interaction.

Let us now consider the case of $\mathcal{N}$ molecules, each of which interact with the quantized electromagnetic field via the electric dipole moment.

\section{MANY MOLECULES}

We consider the case of many molecules with electric dipole moments interacting with electromagnetic field modes whose minimum wavelength $\lambda_{\min }$ is large on the scale of the molecular size $L$; i.e. $\lambda_{\min }>L$ as shown in Fig.1 below. 


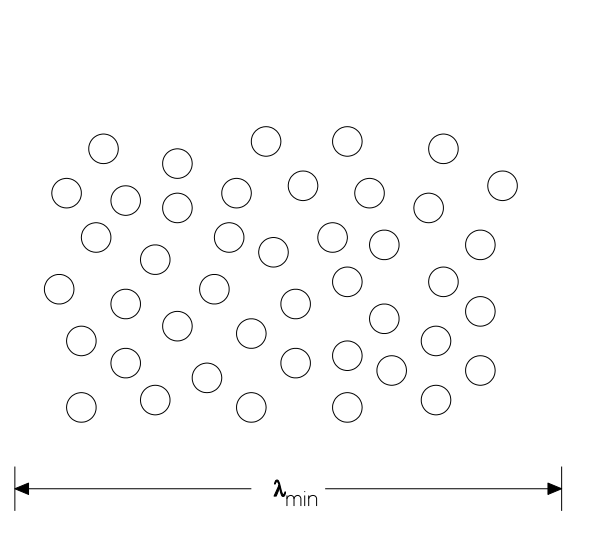

FIG. 1. Shown are many molecules of small size $L$ when compared with the minimum photon wavelength $\lambda_{\min }$.

The Hamiltonian for $\mathcal{N}$ molecules plus the electromagnetic field reads

$$
H=H_{\text {rad }}+\sum_{j=1}^{\mathcal{N}} H_{m o l ; j}(\mathbf{A})+\sum_{1 \leq j<i \leq \mathcal{N}} V_{i j} .
$$

The radiation Hamiltonian in the Coulomb gauge

$$
\operatorname{div} \mathbf{A}(\mathbf{r})=0
$$

is given by

$$
H_{\text {rad }}=\frac{1}{8 \pi} \int\left(\mathbf{E}^{2}+\mathbf{B}^{2}\right) d^{3} \mathbf{r} .
$$

The internal $j^{\text {th }}$ molecular Hamiltonian is given by

$$
\begin{gathered}
H_{m o l ; j}(\mathbf{A})= \\
\sum_{a=1}^{N} K_{a}\left(\mathbf{p}_{(a ; j)}-\frac{q_{a}}{c} \mathbf{A}\left(\mathbf{R}_{j}\right)\right)+V\left(\mathbf{r}_{(1 ; j)}, \ldots, \mathbf{r}_{(N ; j)}\right),
\end{gathered}
$$

where $\mathbf{R}_{j}$ is the central position of the $j^{t h}$ molecule.

The internal Hamiltonian of each molecule contains the internal Coulomb potentials. In the last term on the right hand side of Eq.(21), $V_{i j}$ describes the Coulomb interactions between two different molecules. The long ranged part of this potential is of the conventional dipoledipole interaction form

$$
V_{i j}^{\text {dipole }}=\mu_{i} \cdot\left(\frac{\left|\mathbf{R}_{i j}\right|^{2} \mathbf{1}-3 \mathbf{R}_{i j} \mathbf{R}_{i j}}{\left|\mathbf{R}_{i j}\right|^{5}}\right) \cdot \mu_{j}
$$

where $\mathbf{R}_{i j}=\mathbf{R}_{i}-\mathbf{R}_{j}$.

The commutation relations of the quantum electrodynamic fields in the Coulomb gauge are given by

$$
\left[\mathbf{E}(\mathbf{r}), \mathbf{A}\left(\mathbf{r}^{\prime}\right)\right]=4 \pi i \hbar c \boldsymbol{\Delta}\left(\mathbf{r}-\mathbf{r}^{\prime}\right)
$$

where the transverse delta function is defined as

$$
\boldsymbol{\Delta}(\mathbf{r})=\int(\mathbf{1}-\hat{\mathbf{k}} \hat{\mathbf{k}}) e^{i \mathbf{k} \cdot \mathbf{r}}\left(\frac{d^{3} \mathbf{k}}{(2 \pi)^{3}}\right)
$$

Equivalently

$$
\boldsymbol{\Delta}(\mathbf{r})=\int\left(\frac{|\mathbf{k}|^{2} \mathbf{1}-\mathbf{k} \mathbf{k}}{|\mathbf{k}|^{2}}\right) e^{i \mathbf{k} \cdot \mathbf{r}}\left(\frac{d^{3} \mathbf{k}}{(2 \pi)^{3}}\right),
$$

so that

$$
\boldsymbol{\Delta}(\mathbf{r})=\left(\frac{1}{4 \pi}\right)\left(\nabla \nabla-\mathbf{1} \nabla^{2}\right) \frac{1}{|\mathbf{r}|} .
$$

Explicitly 29]

$$
\boldsymbol{\Delta}(\mathbf{r})=\left(\frac{2}{3}\right) \mathbf{1} \delta(\mathbf{r})+\left(\frac{1}{4 \pi}\right)\left(\frac{3 \mathbf{r r}-|\mathbf{r}|^{2} \mathbf{1}}{|\mathbf{r}|^{5}}\right)
$$

Eqs.(25) and (30) imply

$$
V_{i j}^{\text {dipole }}=-4 \pi \mu_{i} \cdot \boldsymbol{\Delta}\left(\mathbf{R}_{i j}\right) \cdot \mu_{j} \text { for } \mathbf{R}_{i j} \neq 0 .
$$

The transverse distribution function $\Delta(\mathbf{r})$ removes the longitudinal part of a vector field. For example, if

$$
\tilde{\mathbf{P}}(\mathbf{r})=\sum_{j=1}^{\mathcal{N}} \mu_{j} \delta\left(\mathbf{r}-\mathbf{R}_{j}\right)
$$

represents polarization, i.e. the molecular dipole moment per unit volume, then

$$
\mathbf{P}(\mathbf{r})=\int \boldsymbol{\Delta}\left(\mathbf{r}-\mathbf{r}^{\prime}\right) \cdot \tilde{\mathbf{P}}\left(\mathbf{r}^{\prime}\right) d^{3} \mathbf{r}^{\prime}
$$

represents the transverse part of the polarization.

Now, consider the unitary operator

$$
\mathcal{S}=\exp \left(\frac{i}{\hbar c} \int \mathbf{A}(\mathbf{r}) \cdot \tilde{\mathbf{P}}(\mathbf{r}) d^{3} \mathbf{r}\right)
$$

Eq.(26), (33) and (34) imply

$$
\mathcal{S}^{\dagger} \mathbf{E}(\mathbf{r}) \mathcal{S}=\mathbf{E}(\mathbf{r})-4 \pi \mathbf{P}(\mathbf{r})
$$

so that the radiation Hamiltonian of Eq.(23) reads

$$
\mathcal{S}^{\dagger} H_{\text {rad }} \mathcal{S}=\frac{1}{8 \pi} \int\left(|\mathbf{E}-4 \pi \mathbf{P}|^{2}+|\mathbf{B}|^{2}\right) d^{3} \mathbf{r} .
$$

Eqs.(17), (23), (31)-(33) and (36) imply that

$$
\begin{array}{r}
\mathcal{S}^{\dagger} H_{\text {rad }} \mathcal{S}=H_{\text {rad }}-\int \mathbf{E} \cdot \mathbf{P} d^{3} \mathbf{r} \\
+\sum_{j=1}^{\mathcal{N}} W_{\text {mol } ; j}-\sum_{1 \leq i<j \leq \mathcal{N}} V_{i j}^{\text {dipole }} .
\end{array}
$$

It is useful to define the "screened" (or short ranged) intermolecular Coulomb potential with the long ranged dipole-dipole potential subtracted 


$$
\tilde{V}_{i j}=V_{i j}-V_{i j}^{\text {dipole }}
$$

Finally, the unitary transformation removes the vector potential from the internal degrees of freedom of a molecule as in the above Sec.II; i.e. in the dipole interaction limit

$$
\mathcal{S}^{\dagger} H_{m o l ; j}(\mathbf{A}) \mathcal{S}=H_{m o l ; j}(\mathbf{A}=0) .
$$

The "grand finale" of the algebraic ceremony of this section is the assertion that total transformed Hamiltonian

$$
\mathcal{H}=\mathcal{S}^{\dagger} H \mathcal{S}
$$

which for $\mathcal{N}$ molecules takes the form

$$
\begin{aligned}
\mathcal{H}= & \frac{1}{8 \pi} \int\left(\mathbf{E}^{2}+\mathbf{B}^{2}\right) d^{3} \mathbf{r}+\sum_{1 \leq i<j \leq \mathcal{N}} \tilde{V}_{i j} \\
& +\sum_{j=1}^{\mathcal{N}} \mathcal{H}_{m o l ; j}-\int \mathbf{E} \cdot \mathbf{P} d^{3} \mathbf{r},
\end{aligned}
$$

where the internal molecular Hamiltonian

$$
\mathcal{H}_{m o l ; j}=H_{m o l ; j}(\mathbf{A}=0)+W_{m o l ; j}
$$

is independent of the transverse A. The central Eq.(41) of this section follows from Eqs.(17), (24), (37)-(39) and (40).

\section{DICKE-PREPARATA MODEL}

The Hamiltonian in Eq.(41) has four parts: (i) a sum of intramolecular Hamiltonians describing the internal kinetic and Coulomb energies of each molecule, (ii) a sum of screened (short ranged) intermolecular Coulomb potentials between neighboring molecules, (iii) the radiation field energy and (iv) a linear coupling between the dipole moment per unit volume and the electric field. Within the dipole approximation, Eq.(41) is exact but not yet amenable to rigorous mathematical solution. For the purpose of obtaining reasonable answers for (say) the free energy, further simplifications must be made.

For the Dicke-Preparata model, the approximations are as follows: (i) The sum of intramolecular Hamiltonians is modeled by a sum of "two-level molecules", each described by Pauli matrices $\left(\sigma_{x ; j}, \sigma_{y ; j}, \sigma_{z ; j}\right)$;

$$
\sum_{j=1}^{\mathcal{N}} \mathcal{H}_{m o l ; j} \Rightarrow \frac{\varepsilon}{2} \sum_{j=1}^{\mathcal{N}} \sigma_{z ; j}
$$

(ii) The sum of screened intermolecular Coulomb potentials between neighboring molecules is neglected

$$
\sum_{1 \leq i<j \leq \mathcal{N}} \tilde{V}_{i j} \Rightarrow 0
$$

(iii) The radiation field energy is modeled by a single photon oscillator mode

$$
\frac{1}{8 \pi} \int\left(\mathbf{E}^{2}+\mathbf{B}^{2}\right) d^{3} \mathbf{r} \Rightarrow \hbar \omega_{0} b^{\dagger} b .
$$

(iv) The linear coupling between the dipole moment per unit volume and the electric field is modeled by the resonant photon creation and annihilation terms

$$
-\int \mathbf{E} \cdot \mathbf{P} d^{3} \mathbf{r} \Rightarrow \tilde{\mu} \sqrt{\frac{2 \pi \hbar \omega_{0}}{\Omega}} \sum_{j=1}^{\mathcal{N}}\left(b \sigma_{+; j}+b^{\dagger} \sigma_{-; j}\right),
$$

where $2 \sigma_{ \pm ; j}=\sigma_{x ; j} \pm i \sigma_{y ; j}$ and $\tilde{\mu}$ is the excitation matrix element of the electric dipole operator of a single molecule. Putting the model replacement Eqs.(43)-(46) into Eq.(41) yields the Dicke-Preparata model

$$
\mathcal{H} \Rightarrow \mathcal{H}_{D P}
$$

where

$$
\begin{gathered}
\mathcal{H}_{D P}=\varepsilon S_{z}+\hbar \omega_{0} b^{\dagger} b+\tilde{\mu} \sqrt{\frac{2 \pi \hbar \omega_{0}}{\Omega}}\left(b S_{+}+b^{\dagger} S_{-}\right), \\
S_{z}=\frac{1}{2} \sum_{j=1}^{\mathcal{N}} \sigma_{z ; j},
\end{gathered}
$$

and

$$
S_{ \pm}=\frac{1}{2} \sum_{j=1}^{\mathcal{N}}\left(\sigma_{x ; j} \pm i \sigma_{y ; j}\right)
$$

The Dicke-Preparata model may be written in terms of the oscillator coordinate $Q$ and momentum $P$ using

$$
b=\frac{\left(P-i \omega_{0} Q\right)}{\sqrt{2 \hbar \omega_{0}}}, \quad b^{\dagger}=\frac{\left(P+i \omega_{0} Q\right)}{\sqrt{2 \hbar \omega_{0}}},
$$

yielding

$$
\mathcal{H}_{D P}^{\prime}=\left(\frac{P^{2}+\omega_{0}^{2} Q^{2}}{2}\right)+\mathbf{h} \cdot \mathbf{S}
$$

where the vector $\mathbf{h}$ is given by

$$
\mathbf{h}=\tilde{\mu} \sqrt{\frac{4 \pi}{\Omega}}\left(P \mathbf{i}+\omega_{0} Q \mathbf{j}\right)+\varepsilon \mathbf{k} .
$$

The free energy $\mathcal{F}$ of the model is determined by

$$
e^{-\mathcal{F} / k_{B} T}=\operatorname{Tr} e^{-\mathcal{H}_{D P}^{\prime} / k_{B} T} .
$$

Here, the trace over the oscillator degree of freedom may be taken as being classical with virtually zero error in the $\operatorname{limit} \mathcal{N} \rightarrow \infty$; i.e.

$$
e^{-\mathcal{F} / k_{B} T}=\int_{-\infty}^{\infty} \int_{-\infty}^{\infty} \operatorname{tr} e^{-\mathcal{H}_{D P}^{\prime} / k_{B} T}\left(\frac{d P d Q}{2 \pi \hbar}\right) .
$$


Since

$$
\operatorname{tr} e^{-\mathbf{h} \cdot \mathbf{S} / k_{B} T}=\left\{2 \cosh \left(\frac{|\mathbf{h}|}{2 k_{B} T}\right)\right\}^{\mathcal{N}},
$$

and since Eq.(53) implies

$$
|\mathbf{h}|^{2}=\varepsilon^{2}+\left(\frac{4 \pi \tilde{\mu}^{2}}{\Omega}\right)\left(P^{2}+\omega_{0}^{2} Q^{2}\right),
$$

it follows from Eqs.(52), (53), and (55)-(57) that

$$
e^{-\mathcal{F} / k_{B} T}=\int_{-\infty}^{\infty} \int_{-\infty}^{\infty} e^{-\mathcal{G} / k_{B} T}\left(\frac{d P d Q}{2 \pi \hbar}\right),
$$

where

$$
\frac{\mathcal{G}}{\mathcal{N}}=w-k_{B} T \ln \left(2 \cosh \left\{\frac{\sqrt{\varepsilon^{2}+8 \pi \tilde{\mu}^{2} n w}}{2 k_{B} T}\right\}\right),
$$

the oscillator energy per molecule is

$$
w=\left(\frac{P^{2}+\omega_{0}^{2} Q^{2}}{2 \mathcal{N}}\right),
$$

and the number of molecules per unit volume $n$ is

$$
n=(\mathcal{N} / \Omega) .
$$

In the thermodynamic limit of Eqs.(58)-(61), the minimum free energy principle is that

$$
\lim _{\mathcal{N} \rightarrow \infty}(\mathcal{F} / \mathcal{N})=\inf _{(0<w<\infty)} f(w, n, T),
$$

where

$$
f(w, n, T)=w-k_{B} T \ln (2 \cosh \chi(w, n, T)),
$$

and

$$
\chi(w, n, T)=\frac{\sqrt{\varepsilon^{2}+8 \pi \tilde{\mu}^{2} n w}}{2 k_{B} T} .
$$

Let $\bar{w}(n, T) \geq 0$ represent the mean oscillator energy per molecule as a function of the molecular density $n$ and the temperature $T$. If $\bar{w}>0$, then the system is in a super-radiant phase. If $\bar{w}=0$, then the system is in a normal (incoherent radiation) phase. The free energy per molecule $f(w, n, T)$ is at a minimum for $w=$ $\bar{w}$. Setting $(\partial f / \partial w)_{n T}=0$ yields the following implicit function equation for $\bar{w}$ :

$$
\left(\frac{k_{B} T \chi(\bar{w}, n, T)}{\pi \tilde{\mu}^{2} n}\right)=\tanh \chi(\bar{w}, n, T) .
$$

The phase diagram in the $(n, T)$ plane follows from setting $\bar{w}\left(n, T_{c}\right)=0$ and solving for the critical temperature $T_{c}(n)$ as a function of density. The super-radiant phase exists for $T<T_{c}(n)$ and the normal phase exists for $T>T_{c}(n)$. The critical temperature $T_{c}\left(n \leq n_{c}\right)=0$ where the critical density

$$
n_{c}=\left(\frac{\varepsilon}{2 \pi \tilde{\mu}^{2}}\right) .
$$

Analytically,

$$
k_{B} T_{c}\left(n>n_{c}\right)=\left\{\frac{\varepsilon}{\ln \left(\left(n+n_{c}\right) /\left(n-n_{c}\right)\right)}\right\} .
$$

A plot of the phase diagram is shown below.

\section{Phase Diagram}

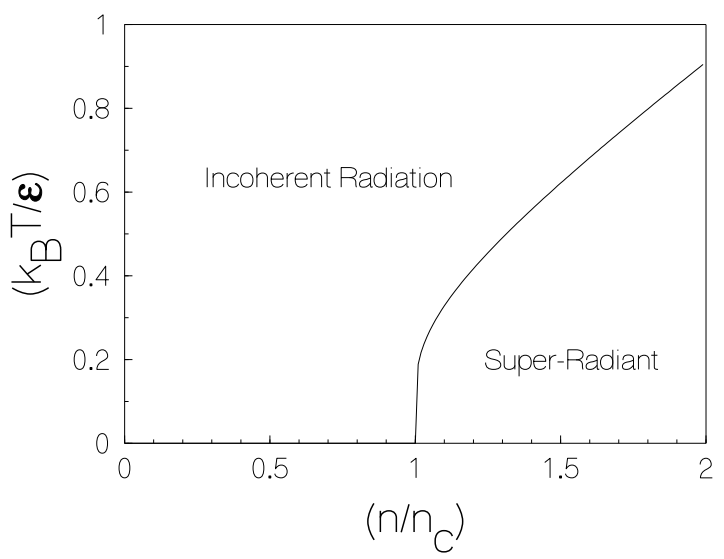

FIG. 2. Shown is the phase diagram in the $(n, T)$-plane, where $n_{c}$ is defined in Eq.(66). The two phases are separated by the critical temperature curve $\left(k_{B} T_{c}(n) / \varepsilon\right)$.

\section{CONCLUSIONS}

The doubts about the physical reality of the superradiant phases were discussed in great detail by $\mathrm{I}$. Bialynicki-Birula and K. Rzazewski [23], who came to the remarkable (and dubious) conclusion that the electric dipole-photon interaction could be gauged away. This incorrect conclusion was based on using a vector potential which is independent of both space and time. It is no wonder that such a pure "gauge field" had zero effect on the thermodynamic equations of state.

In reality, the transverse electric field in the Coulomb gauge should be calculated using either

$$
\mathbf{E}=-\frac{1}{c} \frac{\partial \mathbf{A}}{\partial t}, \quad \text { (Heisenberg or Classical Picture), }
$$

or

$$
\mathbf{E}=-\left(\frac{i}{\hbar c}\right)[H, \mathbf{A}], \quad \text { (Schrodinger Picture) }
$$


The classical canonical transformation Eq.(12) of I. Bialynicki-Birula and K. Rzazewski [23] (in the dipole approximation) should have been properly performed as in our Eqs.(5) and (6). The last term on the right hand side of both our Eqs.(5) and (6) were incorrectly omitted in Eq.(12) of I. Bialynicki-Birula and K. Rzazewski [23]. This incorrect classical canonical transformation eliminated the electric field $\mathbf{E}$ and thereby the electric dipole-photon interaction.

A proper procedure is to employ a fully quantum electrodynamic framework to obtain a non-trivial electric field $\mathbf{E}$ before going to the classical electrodynamic oscillator trace evaluation. From this rigorous Schrödinger picture point of view, Eq.(12) of I. Bialynicki-Birula and K. Rzazewski [23] is still incorrect because Eqs.(B2-B3) in our Appendix B Eqs.(B2-B3) were not taken into account in that work [23].

In any or all pictures, the electric dipole-photon interaction cannot be described by gauging away the electric field $\mathbf{E}$. When the Hamiltonian is expressed in terms of $\mathbf{E}$ and $\mathbf{B}$, it is then properly gauge invariant and non-trivial changes very surely appear in the thermal equations of state. For example, the one-photon loop corrections to the equations of state have long been known to produce Casimir-van der Waals forces in condensed matter. Such forces cannot possibly be gauged away and I. BialynickiBirula and K. Rzazewski [23] are thereby in error. A further error is found in K. Rzazewski, K. Wodkiewicz and W. Zakowicz [20], where it is asserted that the superradiant phase transition is null and void due to quadratic coupling in the photon creation and annihilation operators. Had the canonical transformation been carried out correctly, as in the present work, then it would have been noted that the coupling between the electric dipole moment and the photons in the gauge invariant Eq.(41) is strictly linear. The super-radiant phase transition remains intact.

Preparata has recently proposed several examples of super-radiant phases in a recent review 30]. Water is perhaps the most interesting example [1 31. In future work it would appear interesting to relate the super-radiant transition to an instability of the incoherent radiation phase which produces the conventional theoretical and experimental QED Casimir effects. QED effects at the one photon loop level have long proved their importance in the understanding of the long ranged intermolecular forces 29].

\section{MATHEMATICAL APPENDIX A}

Starting from the Schrödinger equation

$$
i \hbar\left(\frac{d|\phi(t)\rangle}{d t}\right)=H(t)|\phi(t)\rangle
$$

one seeks a solution of the form

$$
|\phi(t)\rangle=U(t)|\psi(t)\rangle
$$

where $U^{\dagger}(t)=U^{-1}(t)$. Putting Eq.(A2) into Eq.(A1) yields

$$
\begin{gathered}
i \hbar U(t)\left(\frac{d|\psi(t)\rangle}{d t}\right)+i \hbar\left(\frac{d U(t)}{d t}\right)|\psi(t)\rangle \\
=H(t) U(t)|\psi(t)\rangle,
\end{gathered}
$$

or equivalently

$$
i \hbar\left(\frac{d|\psi(t)\rangle}{d t}\right)=\mathcal{H}(t)|\psi(t)\rangle
$$

where

$$
\mathcal{H}(t)=U^{\dagger}(t) H(t) U(t)-i \hbar U^{\dagger}(t) \frac{d U(t)}{d t} .
$$

Eq.(A5) is the required time dependent canonical transformation.

\section{MATHEMATICAL APPENDIX B}

From Eqs.(1) and (14) it follows that

$$
S^{\dagger} \mathbf{p}_{a} S=\mathbf{p}_{a}+\frac{q_{a}}{c} \mathbf{A}
$$

Eqs.(B1) and (11) then imply

$$
S^{\dagger} H_{m o l}(\mathbf{A}) S=H_{m o l}(\mathbf{A}=0) .
$$

Thus, the vector potential in the dipole interaction theory may be "gauged away". However, the dipole interaction returns to the full Hamiltonian since Eqs.(9) and (14) imply that

$$
S^{\dagger} a_{k} S=a_{k}+i\left(\mathbf{e}_{k} \cdot \mu\right) \sqrt{\frac{2 \pi}{\Omega \hbar \omega_{k}}}
$$

and

$$
S^{\dagger} a_{k}^{\dagger} S=a_{k}^{\dagger}-i\left(\mathbf{e}_{k}^{*} \cdot \mu\right) \sqrt{\frac{2 \pi}{\Omega \hbar \omega_{k}}} .
$$

From Eqs.(7), (B2) and (B3) it follows that

$$
S^{\dagger} H_{\text {rad }} S=H_{\text {rad }}-\mu \cdot \mathbf{E}+W_{\text {mol }}
$$

where the operator electric field $\mathbf{E}$ is given in Eq.(10), and the molecular self energy $W_{m o l}$ is given in Eq.(17). Thus the same canonical transformation which gauges away the vector potential in the molecule Hamiltonian, brings back the electric dipole interaction $-\mu \cdot \mathbf{E}$ when applied to the radiation Hamiltonian via Eq.(B4). Finally, from Eqs.(B2) and (B4) it follows that

$$
\begin{gathered}
S^{\dagger}\left(H_{r a d}+H_{m o l}(\mathbf{A})\right) S= \\
H_{r a d}+H_{m o l}(\mathbf{A}=0)+W_{m o l}-\mu \cdot \mathbf{E}
\end{gathered}
$$


and Eqs.(16) and (18) then follows from Eq.(B5). The proof of Eq.(18) has been completed.

\section{ACKNOWLEDGMENT}

We wish to thank Dr. Flavio Fontana for an initial discussion of this problem.

[1] R.H. Dicke, Phys. Rev. 93, 99 (1954).

[2] K. Hepp and E.H. Lieb, Phys. Rev. A8, 2517 (1973).

[3] K. Hepp and E.H. Lieb, Ann. Phys. 76, 360 (1973).

[4] E. Del Giudice, G. Preparata and G. Vitiello Phys. Rev. Lett. 61, 1085 (1988).

[5] G. Preparata, "Quantum Field theory of Super Radiance" in Problems of Fundamental Modern Physics, Eds. R. Cherubini, P. Dalpiaz and B. Minetti, World Scientific, Singapore (1990).

[6] G. Preparata, Phys. Rev. A38, 233 (1988).

[7] C.P. Enz, Helv. Phys. Acta 70, 141 (1997).

[8] S. Takeno and M. Nagashima, Prog. Theor. Phys. 57, 1507 (1977).

[9] Y.K. Wong and F.T. Hioe, Phys. Rev. A7, 831 (1973).

[10] H.J. Carmichel, C.W. Gardiner and D.F. Walls, Phys. Lett. A46, 47 (1973).

[11] G. Vertogem and A.S. De Vires, Phys. Lett. A48, 451 (1974).

[12] F.T. Hioe, Phys. Rev. A8, 1440 (1973).

[13] Y.A. Kudenko, A.P. Slivinsky and G.M. Zaslavsky, Phys. Lett. A50, 411 (1975).

[14] B.M. Pimentel and A.H. Zimerman Phys. Lett. A53, 200 (1975).

[15] G.M. Zaslavsky, Y.A. Kudenko and A.P. Slivinsky Sov. Phys. Solid State 16, 2197 (1975).

[16] R. Gilmore and C.M. Bowden, Phys. Rev. A13, 1898 (1976).

[17] R. Gilmore and C.M. Bowden, J. Math. Phys. 17, 1617 (1976).

[18] A.A.S. Brito and W.F. Wreszinski, Helv. Phys. Acta 50, 373 (1977).

[19] A. Klemm and V.A. Zagrebnov, Physica A36, 400 (1977).

[20] K. Rzazewski, K. Wodkiewicz and W. Zakowicz, Phys. Rev. Lett. 35, 432 (1975).

[21] K. Rzazewski and K. Wodkiewicz, Phys. Rev. A13, 1967 (1976).

[22] K. Rzazewski, K. Wodkiewicz and W. Zakowicz, Phys. Lett. A58, 211 (1976).

[23] I. Bialynicki-Birula and K. Rzazewski, Phys. Rev. A19, 301 (1979).

[24] K. Gawedzki and K. Rzazewski, Phys. Rev. A23, 2134 (1981).

[25] R.G. Woolley, J. Phys. Lett. A9, L15 (1976).

[26] R.G. Woolley, Adv. Chem. Phys. 33, 153 (1975).

[27] E.A. Power and S. Zienau, Phil. Trans. R. Soc. A251, 427 (1959).
[28] G. Preparata, E. Del Giudice and R. Mele, Mod. Phys. Lett. B7, 1851 (1993).

[29] D.P. Craig and T. Thirunamachandran, "Molecular Quantum Electrodynamics", p 56, Dover Publications, New York (1998).

[30] G. Preparata, "QED Coherence in Matter", World Scientific, Singapore (1995).

[31] E. del Giudice and G. Preparata "A New QED Picture of Water" in "Macroscopic Quantum Coherence" Edited by E. Sassaroli, Y. Srivastava, J. Swain and A. Widom, World Scientific, Singapore (1998). 